\title{
Objective assessment of blood and lymphatic vessel invasion and association with macrophage infiltration in cutaneous melanoma
}

Sarah J Storr ${ }^{1,5}$, Sabreena Safuan ${ }^{1,5}$, Angana Mitra ${ }^{2}$, Faye Elliott ${ }^{2}$, Christopher Walker ${ }^{2}$, Mark J Vasko ${ }^{3}$, Bernard $\mathrm{Ho}^{3}$, Martin Cook ${ }^{4}$, Rabab AA Mohammed ${ }^{3,6}$, Poulam M Patel ${ }^{1}$, Ian O Ellis ${ }^{3}$, Julia A Newton-Bishop ${ }^{2}$ and Stewart G Martin ${ }^{1}$

${ }^{1}$ Academic Oncology, University of Nottingham, School of Molecular Medical Sciences, Nottingham University Hospitals NHS Trust, City Hospital Campus, Nottingham, UK; ${ }^{2}$ Section of Epidemiology and Biostatistics, Leeds Institute of Molecular Medicine, University of Leeds, St James's University Hospital, Leeds, UK; ${ }^{3}$ Department of Histopathology, University of Nottingham, School of Molecular Medical Sciences, Nottingham University Hospitals NHS Trust, Nottingham, UK and ${ }^{4}$ Department of Histopathology, Royal Surrey County Hospitals NHS Trust, Guildford, UK

The aims of this study were to investigate the role of vascular invasion (blood and lymphatic), vessel density and the presence of tumour-associated macrophages as prognostic markers in 202 cutaneous melanoma patients. Sections of primary melanoma were stained with lymphatic-specific antibody D2-40 to assess lymphatic vessel invasion and density in intratumoural and peritumoural areas; an antibody against endothelial marker CD34 was used to determine blood vessel invasion and density, and an antibody against CD68 was used to determine macrophage counts. Immunohistochemically determined vascular invasion (combined blood and lymphatic) was compared with that determined using haematoxylin and eosin (H\&E) staining. The use of immunohistochemistry increased detection of vascular invasion from $8-30 \%$ of patients, and histological exam of H\&E-stained tissue was associated with a false positive rate of $64 \%$. Lymphatic vessel invasion occurred at a much higher frequency than blood vessel invasion (27 and $4 \%$ of patients, respectively). Although immunohistochemically detected vessel invasion was significantly associated with histological markers of adverse prognosis, such as increased Breslow thickness, ulceration and mitotic rate (all $P<0.001$ ), no associations with relapse-free or overall survival were observed. High macrophage counts were significantly associated with markers of aggressive disease, such as Breslow thickness, ulceration and mitotic rate $(P<0.001, P<0.001, P=0.005$, respectively), and lymphatic vessel invasion and high microvessel density $(P=0.002$ and $P=0.003$, respectively). These results suggest that vascular invasion is more accurately detected using immunohistochemistry and occurs predominantly via lymphatic vessels. The association of vessel characteristics with histological characteristics of the primary melanoma provides evidence for their biological importance in melanoma, but that they were not associated with clinical outcome attests to the value of existing histological prognostic biomarkers. We note that a high macrophage count may be associated with neovascularisation and primary tumour growth, and may also promote invasion through lymphatic vessels. Modern Pathology (2012) 25, 493-504; doi:10.1038/modpathol.2011.182; published online 11 November 2011

Keywords: D2-40; melanoma; tumour associated macrophages; vascular invasion; vessel density

Correspondence: Dr SG Martin, Academic Oncology, University of Nottingham, School of Molecular Medical Sciences, Nottingham University Hospitals NHS Trust, City Hospital Campus, Nottingham, NG5 1PB, UK.

E-mail: stewart.martin@nottingham.ac.uk

${ }^{5}$ These authors contributed equally to this work.

${ }^{6}$ Current address: Department of Pathology, Faculty of Medicine, Assiut University, Assiut, Egypt.

Received 26 July 2011; revised 5 October 2011; accepted 5 October 2011; published online 11 November 2011
Cutaneous melanoma is the most potentially lethal form of skin cancer and a number of poor prognostic factors have been identified, such as increased Breslow thickness, tumour ulceration, increased mitotic rate and positive sentinel node biopsy. ${ }^{1}$ These measures are incorporated into the American Joint Committee on Cancer staging system, which explains a large part, but not all, of the variance in survival for melanoma patients. ${ }^{1}$ Vascular invasion, encompassing 
both blood and lymphatic vessel invasion, is an important characteristic of various tumour types and is an independent prognostic factor in certain cancers, such as breast cancer. ${ }^{2}$ Tumour cells have the ability to spread through lymphatic vessels to lymph nodes, making lymphatic vessels important in the initial metastatic cascade of cancer.

In melanoma, the significance of lymphatic invasion and vessel density have been investigated in a small number of studies using immunohistochemistry as a more sensitive method of assessment, using various specific lymphatic markers such as D2-40 and LYVE-1; however, controversy remains over aspects of its clinical relevance. Low lymphatic vessel density has been shown to be associated with both adverse $^{3}$ and improved patient survival. ${ }^{4,5}$ In addition, high lymphatic vessel density has been associated with metastatic spread ${ }^{6-8}$ but also with the absence of vascular invasion. ${ }^{3}$ Lymphatic vessel invasion has been previously associated with various clinicopathological variables, such as ulceration ${ }^{9}$ and increased Breslow thickness, ${ }^{10,11}$ but also clinical outcome, such as metastases and overall survival., , $11,12^{2}$ Evidence for an association between lymphatic vessel invasion and sentinel node biopsy positivity remains inconclusive, with studies containing varying relatively small numbers of patients (27-96 patients) showing either a significant association ${ }^{9,10,12,13}$ or no association. ${ }^{14,15}$ Haematoxylin and eosin (H\&E)-determined vascular invasion in primary melanoma has been shown to be an independent prognostic factor for melanoma survival ${ }^{16-18}$ and sentinel node biopsy positivity. ${ }^{19}$ The presence of macrophage infiltration of the invasive front of melanoma tumours has been shown to be associated with poor overall survival, ${ }^{20}$ and the presence of intratumoural macrophages are associated with survival in sinonasal melanoma; ${ }^{21}$ however, its presence has not been investigated in association with vascular invasion. Interestingly, metastasis in melanoma xenograft models has recently been associated with microvessel density. ${ }^{22}$

The first aim of this study was to investigate the role of lymphatic and blood vessel invasion, and lymphatic and microvessel density as prognostic biomarkers, using immunohistochemistry in melanoma patients. The second aim of this study was to investigate the topography and characteristics of lymphatic and blood vessels in a large cohort of melanoma patients, and to analyse their association with clinicopathological variables and clinical outcome. Finally, we sought to investigate the role that tumourassociated macrophages may have in these processes by investigating the associations between macrophage counts and the characteristics of the vessels.

\section{Materials and methods}

\section{Patients and Specimens}

Patients with cutaneous melanoma of Breslow thickness $\geqslant 0.75 \mathrm{~mm}$, having undergone sentinel node biopsy, were recruited to a multi-centre casecontrol study designed to identify predictors of sentinel node biopsy positivity and outcome. ${ }^{23}$ This study was conducted on 202 paraffin-embedded archival specimens obtained from patients having had a sentinel node biopsy between 1994 and 2006 at five UK centres. Ethical approval for the study was granted by Leeds (East) REC (06/Q1206/149). Clinical data were taken from patient case notes. The median age of the patients was 53 years, with a range of $14-88$ years; $65 \%(132 / 202)$ had a positive sentinel lymph node biopsy; 49\% (99/202) of the patients were female. Breslow thickness, ulceration, mitotic rate, the presence of microsatellites and histological subtype, were all recorded by one expert melanoma pathologist (MC). A total of $32 \%$ (62 out of 196) of patients relapsed and $22 \%$ of patients died (45 out of 200) within this cohort, and the mean follow-up time was 38 months. Relapse-free survival was calculated from the date of the initial surgery to the first relapse, or from the date of the initial surgery to the last date known to have not relapsed for those censored. Overall survival time was calculated from the date of the initial surgery to death, or from date of the initial surgery to last date known to be alive for those censored.

\section{Immunohistochemistry}

For visualisation of vessels and macrophages, three consecutive formalin-fixed paraffin-embedded archival sections from each specimen were stained with antibodies against CD34 (AbD Serotec, Kidlington, UK) and CD68 (Abcam, Cambridge, UK) to stain blood vessels and macrophages, respectively. Antibody D2-40 (Covance SIGNET, NJ, USA) was used to stain lymphatic vessels. Tissue was stained as previously described. ${ }^{24,25}$ Briefly, 4- $\mu \mathrm{m}$ sections were deparaffinised in histolene, followed by rehydration in ethanol. For CD34 and CD68 antibodies, antigen retrieval was performed in $0.01 \mathrm{~mol} / \mathrm{l}$ sodium citrate buffer (pH6) in a microwave. Endogenous peroxidase activity was blocked in $0.01 \%$ hydrogen peroxide in methanol, and non-specific binding was blocked using normal swine serum (Dako, Glostrup, Denmark). Primary antibodies (CD34 1:500; D2-40 1:100; CD68 1:100) were incubated on the tissue for $1 \mathrm{~h}$ before incubation with biotinylated secondary antibody according to the manufacturer's instructions (StreptABComplex/HRP Duet; Dako). CD34 and D2-40 reactions were developed with $3,3^{\prime}$ diaminobenzidine as the chromogenic peroxidase substrate, and CD68 reactions were developed with permanent red substrate (Dako) to distinguish macrophages from melanocytes. Sections were counterstained with Gills formula Haematoxylin (Vector Laboratories, Peterborough, UK), dehydrated and fixed before mounting with DPX, or Glycergel mounting medium for CD68-stained sections. Sections of tonsil or placental tissue were 
included as controls, where primary antibody was omitted for negative controls. In addition, a further consecutive section was stained using H\&E for comparison against immunostained tissues.

\section{Assessment of Microvessel Density and Macrophage Count}

Microvessel density and the number of macrophages in each tissue section were counted using the 'hotspot' Chalkley 25-point method in three areas, with an average value used for analysis as previously described. ${ }^{24-26}$ Slides $(20 \%)$ were examined by two independent assessors blinded to clinicopathological data and scores.

\section{Assessment of Lymphatic Vessel Density}

Lymphatic vessel density was assessed by counting all positively stained vessels in the whole section as previously described. ${ }^{27}$ Briefly, the sections were divided into two areas, intratumoural and peritumoural, and positive vessels were counted across the total tumour area, which was determined in $\mathrm{mm}^{2}$ by approximating tumour size by counting fields of view, each representing a surface area of $154 \mathrm{~mm}^{2}$. Lymphatic vessel density in each area was determined by dividing the sum of lymphatic vessels in that area by the total surface area, presented as vessels per $\mathrm{mm}^{2}$. Total lymphatic vessel density for each specimen is the sum of both intratumoural and peritumoural lymphatic vessel densities. Slides $(30 \%)$ were examined by two independent assessors blinded to clinicopathological data and scores.

\section{Assessment of Vascular Invasion}

H\&E assessment was initially performed to identify those tumours as vascular invasion positive, negative or probable. Using immunohistochemically stained sections, lymphatic vessel invasion was determined as the presence of tumour cells within a D2-40-stained vessel. Blood vessel invasion was determined as the presence of tumour cells in vessels stained with anti-CD34 antibody, but not using D2-40 antibody. Sections with either lymphatic vessel invasion or blood vessel invasion were classed as vascular invasion positive. All probable lymphatic vessel invasions were reviewed by a consultant pathologist (MC and/or IOE). Vascular invasion was categorised as negative, probable and positive, and for analysis, probable invasions were grouped with the positive grouping. The frequency of vascular invasion assessed by immunohistochemistry was compared with $\mathrm{H} \& \mathrm{E}$ staining; false positives were recorded when H\&E-determined vascular invasion was shown to be an artefact by immunohistochemistry; false negatives were reported as negative by $\mathrm{H} \& \mathrm{E}$, but immunohistochemistry revealed vascular invasion to be present. The location of lymphatic vessel invasion was also topographically assessed as within the intratumoural or peritumoural areas.

\section{Statistical Analysis}

Some tumours were not scored due to a lack of peritumoural or missing tissue. To test for associations between vascular invasion, vessel density and clinicopathological variable data, Pearson's $\chi^{2}$-test of association was used, or Fisher's exact test if a cell count was $<5$. Vessel density and macrophage counts were assessed by categorising data as high or low around the median value. Overall survival and relapse-free survival curves were plotted according to the Kaplan-Meier method and assessed further, using multivariate survival analysis using Cox regression. An arbitrary significance level of $1 \%$ was used. Statistical analysis was carried out using STATA version 10 (StataCorp 2007, Stata statistical software: Release 10, College Station, TX: StataCorpLP) and SPSS software version 17.

\section{Results}

\section{Comparison of Immunohistochemistry and H\&E Staining Methodologies}

The presence of vascular invasion (either blood or lymphatic) determined by immunohistochemistry and H\&E staining was directly compared in consecutive sections. In this study, immunohistochemistry detected any vascular invasion (both blood and lymphatic) in $30 \%$ of cases (55 out of 184), whereas $\mathrm{H} \& \mathrm{E}$ staining detected vascular invasion in $8 \%$ of cases (16 out of 199). Of the vascular invasion detected by immunohistochemistry, the majority (85\%; 47 out of 55) was solely via lymphatic vessels, $5 \%$ of tumours had both lymphatic and blood vessel invasion ( 3 out of 55), and $9 \%$ (5 out of 55 ) of tumours had blood vessel invasion alone.

Vessel invasion in the cases, which had both H\&E and immunohistochemistry results available (184 cases), was compared. In this group of cases, 14 patients had vascular invasion evident in sections stained by H\&E, 9 of which were shown to be false positives by immunohistochemistry (64\%). Of the 170 cases negative for vascular invasion determined by $\mathrm{H} \& \mathrm{E}, 50$ were false negative (29\%). Examples of $\mathrm{H} \& \mathrm{E}$ and immunohistochemistry staining are shown in Figure 1.

\section{Relationship between Vascular Invasion and Clinicopathological Data}

Intratumoural, peritumoural, or any lymphatic invasion (a measure resultant from combined intratumoural and peritumoural invasion) was observed in 

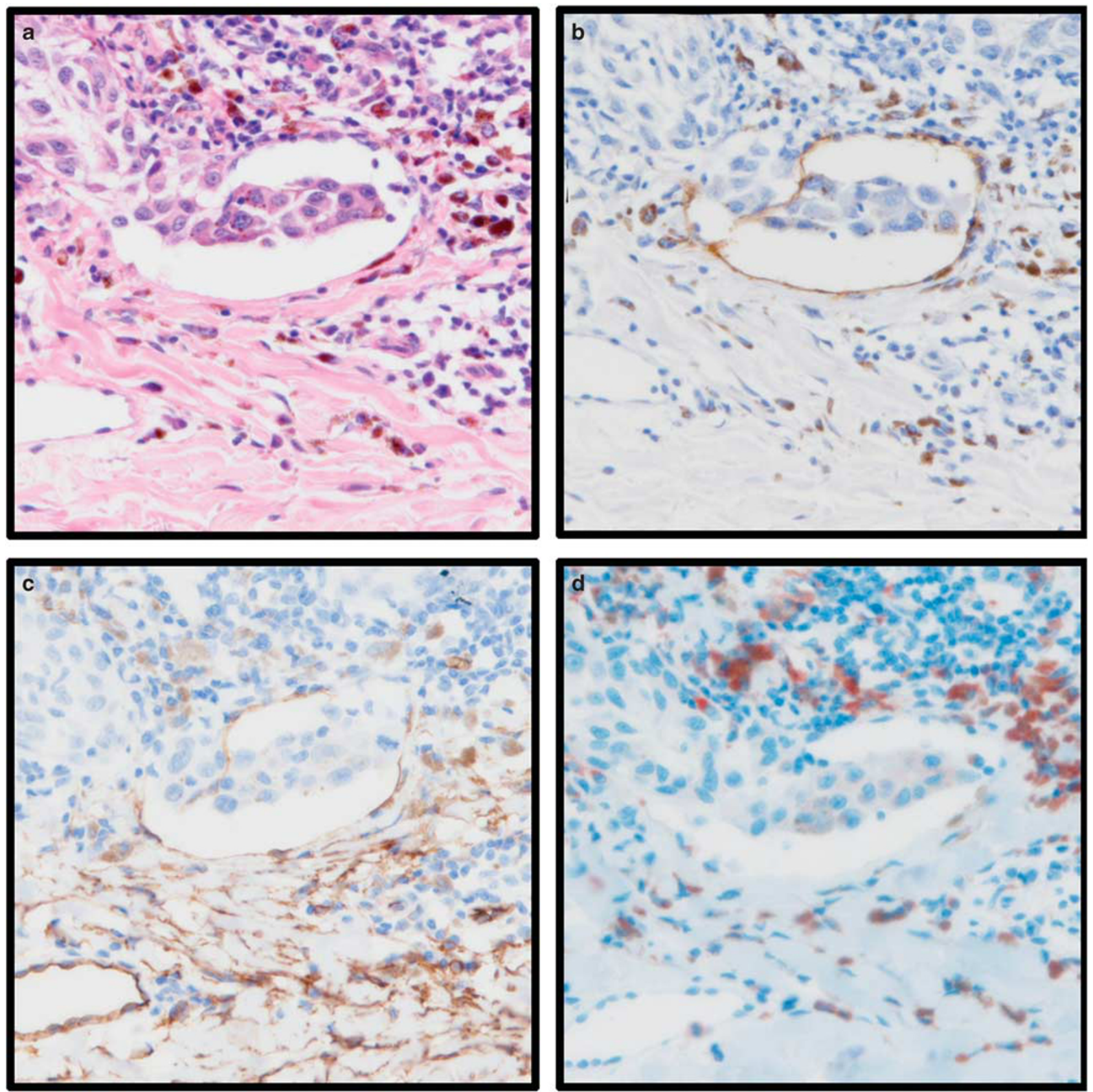

Figure 1 Photomicrographs of tissue stained with D2-40 (lymphatic vessels), anti-CD34 (pan-vascular marker), anti-CD68 (macrophage marker), and haematoxylin and eosin (H\&E) from two tumours (a-d tumour 1, c-h tumour 2). Examples are given of a true positive determined in H\&E-stained tissue (tumour 1) and an example of a false negative in H\&E-stained tissue (tumour 2). Tumour one; (a) vessel invasion determined in H\&E-stained tissue; (b) invasion occurs in D2-40-stained lymphatic vessel; (c) invasion is visible in a vessel stained with anti-CD34; (d) anti-CD68-stained macrophage are observed around the invasion. Tumour 2; (e) vessel invasion not visible in H\&E-stained tissue; (f) invasion occurs in D2-40-stained lymphatic vessel; (g) consecutive section stained with anti-CD34; (h) anti-CD68stained tissue. All images are shown at $\times 20$ magnification.

$17 \%$ (33 out of 191), 15\% (27 out of 181 ), $27 \%$ (50 out of 186) of cases, respectively. Blood vessel invasion occurred in 4\% (8 out of 190) cases, 3 of which also had lymphatic vessel invasion. The presence of intratumoural lymphatic vessel invasion was positively associated with increased stage (Fisher's exact test, $P=0.004$ ), increased Breslow thickness (Fisher's exact test, $P<0.001$ ), the presence of ulceration $\left(\chi^{2}=14.69\right.$, d.f. $\left.=1, P<0.001\right)$, high mitotic rate (Fisher's exact test, $P<0.001$ ) and the presence of microsatellites $\left(\chi^{2}=6.18\right.$, d.f. $=1$, $P=0.01)$ in addition to nodular histological subtype $\left(\chi^{2}=18.94\right.$, d.f. $\left.=2, P<0.001\right)$ (Table 1). Peritumoural lymphatic vessel invasion was positively 

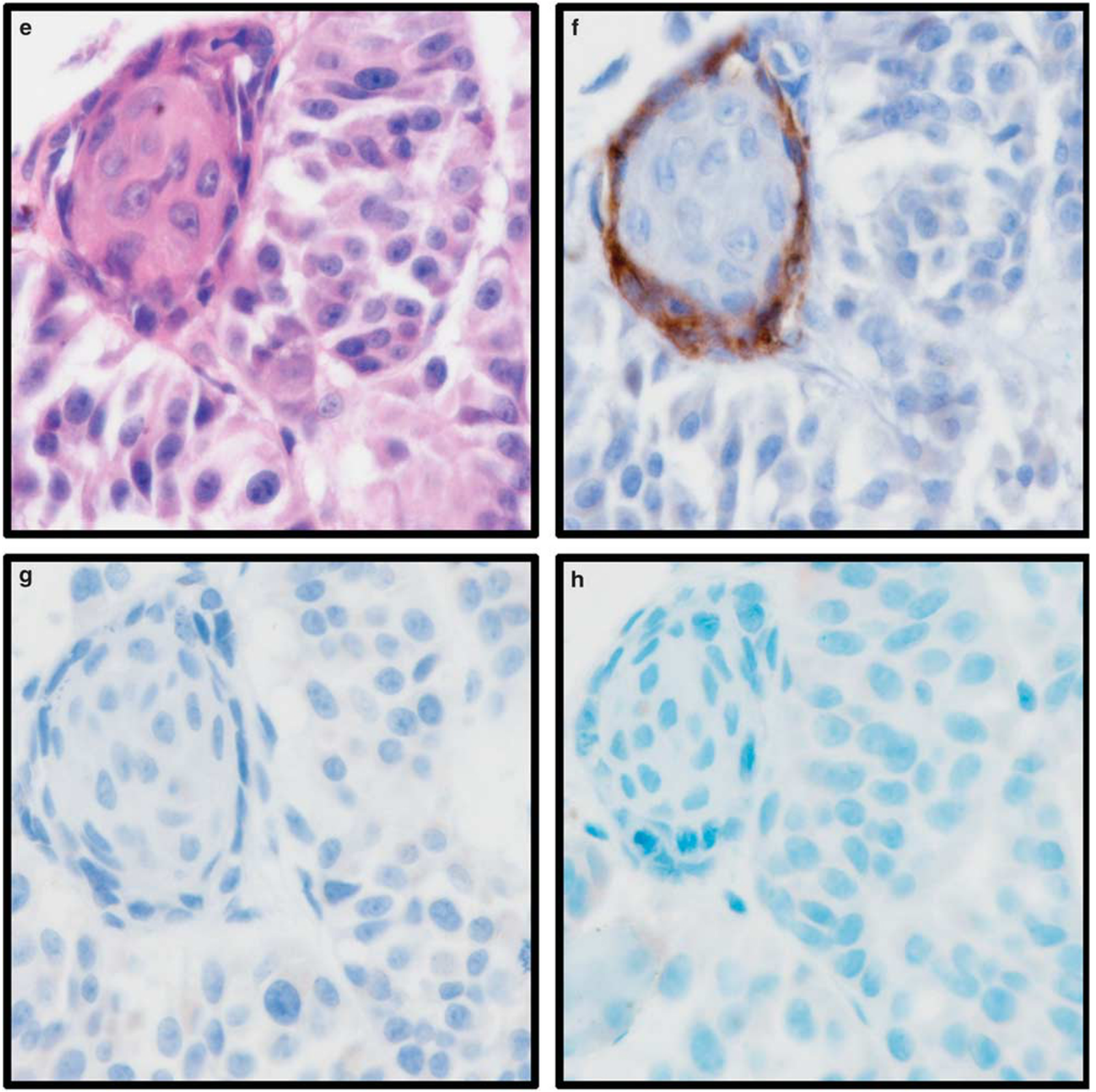

Figure 1 Continued.

associated with increased stage (Fisher's exact test, $P<0.001)$, increased Breslow thickness $\left(\chi^{2}=13.08\right.$, d.f. $=2, P=0.001)$ and the presence of ulceration $\left(\chi^{2}=16.99\right.$, d.f. $\left.=1, P<0.001\right)$, in addition to nodular histological subtype (Fisher's exact test, $P<0.001$; Table 1). Unlike intratumoural lymphatic vessel invasion, peritumoural lymphatic vessel invasion was not associated with mitotic rate or the presence of microsatellites. When analysed as any lymphatic vessel invasion, positive associations were observed with increased stage (Fisher's exact test, $P<0.001)$, increased Breslow thickness $\left(\chi^{2}=29.27, \quad\right.$ d.f. $\left.=2, \quad P<0.001\right)$, the presence of ulceration $\left(\chi^{2}=22.59\right.$, d.f. $\left.=1, P<0.001\right)$, high mitotic rate $\left(\chi^{2}=18.90\right.$, d.f. $\left.=2, P<0.001\right)$, the presence of microsatellites $\left(\chi^{2}=6.64\right.$, d.f. $\left.=1, P=0.01\right)$ and nodular histological subtype $\left(\chi^{2}=28.47\right.$, d.f. $=2$, $P<0.001$; Table 1). Blood vessel invasion was not significantly associated with any clinicopathological variable (Table 2). Immunohistochemically determined vascular invasion (intratumoural, peritumoural lymphatic vessel, or blood vessel invasion), was positively associated with increased stage (Fisher's exact test, $P<0.001$ ), increased Breslow thickness $\left(\chi^{2}=26.58\right.$, d.f. $\left.=2, P<0.001\right)$, the presence of ulceration $\left(\chi^{2}=19.51\right.$, d.f. $\left.=1, P<0.001\right)$ 
Table 1 The table shows the association between intra- and peritumoural lymphatic vessel invasion, any lymphatic invasion, and invasion of any immunohistochemically stained vessel (both blood and lymphatic) with clinicopathological variables in melanoma

\begin{tabular}{|c|c|c|c|c|c|c|c|c|c|c|c|c|}
\hline \multirow[t]{3}{*}{ Category } & \multicolumn{9}{|c|}{ Lymphatic vessel invasion } & \multirow{2}{*}{\multicolumn{3}{|c|}{$\frac{\text { Any vessel invasion }}{\text { (IHC determined) n (\%) }}$}} \\
\hline & \multicolumn{3}{|c|}{ Intratumoural n (\%) } & \multicolumn{3}{|c|}{ Peritumoural n (\%) } & \multicolumn{3}{|c|}{ Any n (\%) } & & & \\
\hline & Absent & Present & P-value & Absent & Present & $\mathrm{P}$-value & Absent & Present & $\mathrm{P}$-value & Absent & Present & P-value \\
\hline \multicolumn{13}{|l|}{ Gender } \\
\hline Female $(n=99)$ & $78(49)$ & $14(42)$ & 0.47 & $76(49)$ & $13(48)$ & 0.91 & $68(50)$ & $23(46)$ & 0.63 & $64(50)$ & $25(45)$ & 0.61 \\
\hline Male $(n=103)$ & $80(51)$ & $19(58)$ & & $78(51)$ & $14(52)$ & & $68(50)$ & $27(54)$ & & $65(50)$ & $30(55)$ & \\
\hline \multicolumn{13}{|c|}{ Sentinal node biopsy (SNB) } \\
\hline Negative $(n=70)$ & $58(37)$ & $8(24)$ & 0.17 & $58(38)$ & $8(30)$ & 0.42 & $53(39)$ & $13(26)$ & 0.10 & $49(38)$ & $14(25)$ & 0.10 \\
\hline Positive $(n=132)$ & $100(63)$ & $25(76)$ & & $96(62)$ & $19(70)$ & & $83(61)$ & $37(74)$ & & $80(62)$ & $41(75)$ & \\
\hline \multicolumn{13}{|l|}{ Relapse status } \\
\hline No $(n=134)$ & $110(71)$ & $18(58)$ & 0.16 & $109(72)$ & $15(56)$ & 0.08 & $98(73)$ & $29(60)$ & 0.10 & $93(73)$ & $31(58)$ & 0.05 \\
\hline Yes $(n=62)$ & $45(29)$ & $13(42)$ & & $42(28)$ & $12(44)$ & & $36(27)$ & $19(40)$ & & $34(27)$ & $22(41)$ & \\
\hline \multicolumn{13}{|l|}{ Staging } \\
\hline IA/IB $(n=43)$ & $38(25)$ & $2(6)$ & $0.004^{*}$ & $39(26)$ & $1(4)$ & $<0.001^{*}$ & $37(28)$ & $3(6)$ & $<0.001^{*}$ & $35(28)$ & $3(6)$ & $<0.001 *$ \\
\hline IIA $(n=11)$ & $10(7)$ & $1(3)$ & & $10(7)$ & $1(4)$ & & $9(7)$ & $2(4)$ & & $8(6)$ & $2(4)$ & \\
\hline $\mathrm{IIB} / \mathrm{IIC}(n=12)$ & $7(5)$ & $4(12)$ & & $6(4)$ & 5 (19) & & $5(4)$ & $6(12)$ & & $5(4)$ & $6(12)$ & \\
\hline IIIA $(n=88)$ & $71(47)$ & $13(39)$ & & $69(47)$ & $8(31)$ & & $62(47)$ & $17(35)$ & & $59(47)$ & $20(38)$ & \\
\hline IIIB $(n=41)$ & $26(17)$ & $13(39)$ & & $24(16)$ & $11(42)$ & & $18(14)$ & $20(42)$ & & $18(14)$ & $21(40)$ & \\
\hline \multicolumn{13}{|l|}{ Number of positive nodes } \\
\hline $0(n=70)$ & $58(37)$ & $8(24)$ & $0.34^{*}$ & $58(38)$ & $8(30)$ & $0.05^{*}$ & $53(39)$ & $13(26)$ & $0.16^{*}$ & $49(38)$ & $14(25)$ & $0.09^{*}$ \\
\hline 1 or $2(n=123)$ & $93(59)$ & $23(70)$ & & $91(59)$ & $15(56)$ & & $78(57)$ & $33(66)$ & & 76 (59) & $36(65)$ & \\
\hline$\geqslant 3(n=9)$ & $7(4)$ & $2(6)$ & & $5(3)$ & $4(15)$ & & $5(4)$ & $4(8)$ & & $4(3)$ & $5(9)$ & \\
\hline \multicolumn{13}{|l|}{ Melanoma site } \\
\hline Trunk $(n=73)$ & $63(40)$ & $9(27)$ & $0.43^{*}$ & $59(38)$ & $8(30)$ & $0.77^{*}$ & $56(41)$ & $13(26)$ & $0.26^{*}$ & $53(41)$ & $16(29)$ & $0.46^{*}$ \\
\hline $\operatorname{Leg}(n=67)$ & $52(33)$ & $11(33)$ & & $48(31)$ & $11(41)$ & & $42(31)$ & $19(38)$ & & $39(30)$ & $21(38)$ & \\
\hline $\operatorname{Arm}(n=45)$ & $32(20)$ & $10(30)$ & & $35(23)$ & $6(22)$ & & $28(21)$ & $14(28)$ & & $28(22)$ & $14(25)$ & \\
\hline Head/neck $(n=17)$ & $11(7)$ & $3(9)$ & & $12(8)$ & $2(7)$ & & $10(7)$ & $4(8)$ & & $9(7)$ & $4(7)$ & \\
\hline \multicolumn{13}{|l|}{ Histological subtype } \\
\hline $\begin{array}{l}\text { Superficial spreading } \\
(n=141)\end{array}$ & $120(82)$ & $15(45)$ & $<\mathbf{0 . 0 0 1}^{*}$ & $121(82)$ & $11(46)$ & $<\mathbf{0 . 0 0 1}^{*}$ & $111(85)$ & $22(47)$ & $<0.001$ & $106(85)$ & $24(48)$ & $<0.001$ \\
\hline Nodular $(n=37)$ & $20(14)$ & $14(42)$ & & $19(13)$ & $11(46)$ & & $13(10)$ & $20(43)$ & & $13(10)$ & $20(40)$ & \\
\hline Other $(n=12)$ & $7(5)$ & $4(12)$ & & $8(5)$ & $2(8)$ & & $6(5)$ & $5(11)$ & & $5(4)$ & $6(12)$ & \\
\hline \multicolumn{13}{|l|}{ Breslow thickness (mm) } \\
\hline$\leqslant 1(n=94)$ & $84(53)$ & $4(12)$ & $<0.001^{*}$ & $82(53)$ & $5(19)$ & 0.001 & $79(58)$ & $8(16)$ & $<0.001$ & $75(58)$ & $10(18)$ & $<0.001$ \\
\hline$>1 \leqslant 2(n=79)$ & $58(37)$ & $17(52)$ & & $55(36)$ & $14(52)$ & & $45(33)$ & $27(54)$ & & $42(33)$ & $30(55)$ & \\
\hline$>2 \leqslant 4(n=29)$ & $16(10)$ & $12(36)$ & & $17(11)$ & $8(30)$ & & $12(9)$ & $15(30)$ & & $12(9)$ & $15(27)$ & \\
\hline \multicolumn{13}{|l|}{ Ulceration } \\
\hline No $(n=145)$ & $121(80)$ & $15(47)$ & $<0.001$ & $119(80)$ & $11(42)$ & $<0.001$ & $109(83)$ & $23(48)$ & $<0.001$ & $103(82)$ & $26(50)$ & $<0.001$ \\
\hline Yes $(n=50)$ & $31(20)$ & $17(53)$ & & $29(20)$ & $15(58)$ & & $22(17)$ & $25(52)$ & & $22(18)$ & $26(50)$ & \\
\hline Mitotic rate $\left(\right.$ per $\left.\mathrm{mm}^{2}\right)$ & & & & & & & & & & & & \\
\hline$<3(n=68)$ & $62(39)$ & $2(6)$ & $<0.001^{*}$ & $57(37.3)$ & $5(18.5)$ & 0.09 & $55(41)$ & $7(14)$ & $<0.001$ & $52(41)$ & $8(15)$ & $<0.001$ \\
\hline $3-7(n=62)$ & $52(33)$ & $6(18)$ & & $47(30.7)$ & $8(29.6)$ & & $43(32)$ & $13(26)$ & & $41(32)$ & $14(25)$ & \\
\hline$>7(n=71)$ & $43(37)$ & $25(76)$ & & $49(32.0)$ & $14(51.9)$ & & $37(27)$ & $30(60)$ & & $35(27)$ & $33(60)$ & \\
\hline Perineural infiltrat & & & & & & & & & & & & \\
\hline No $(n=186)$ & $143(98)$ & $32(100)$ & $1.00^{*}$ & $141(98.6)$ & $25(96.2)$ & $0.40^{*}$ & $124(98)$ & 47 (98) & $1.00^{*}$ & $118(98)$ & $50(98)$ & $1.00^{*}$ \\
\hline Yes $(n=3)$ & $3(2)$ & $0(0)$ & & $2(1.4)$ & $1(3.8)$ & & $2(2)$ & $1(2)$ & & $2(2)$ & $1(2)$ & \\
\hline $\operatorname{Reg}$ & & & & & & & & & & & & \\
\hline No $(n=171)$ & $128(82)$ & $32(97)$ & $0.03^{*}$ & $131(86.2)$ & $19(70.4)$ & 0.04 & $114(85)$ & $41(82)$ & 0.61 & $107(84)$ & $46(84)$ & 0.917 \\
\hline Yes $(n=29)$ & $28(18)$ & $1(3)$ & & $21(13.8)$ & $8(29.6)$ & & $20(15)$ & $9(18)$ & & $20(16)$ & $9(16)$ & \\
\hline Micro & & & & & & & & & & & & \\
\hline No $(n=163)$ & $132(90)$ & $23(74)$ & 0.01 & $126(88.7)$ & $21(80.8)$ & 0.26 & $115(91)$ & $36(77)$ & 0.01 & $110(92)$ & $38(76)$ & 0.006 \\
\hline Yes $(n=25)$ & $14(10)$ & $8(26)$ & & $16(11.3)$ & $5(19.2)$ & & $11(9)$ & $11(23)$ & & $10(8)$ & $12(24)$ & \\
\hline Tumour infiltra & ocytes (T & ILs) & & & & & & & & & & \\
\hline Absent $(n=52)$ & $41(26)$ & $9(28)$ & $0.41^{*}$ & $42(27.6)$ & $5(18.5)$ & $0.23^{*}$ & $36(27)$ & $12(24)$ & $0.19^{*}$ & $32(25)$ & $16(30)$ & $0.19^{*}$ \\
\hline Non-brisk $(n=126)$ & $96(62)$ & $22(69)$ & & $92(60.5)$ & $21(77.8)$ & & $81(60)$ & $35(71)$ & & $79(62)$ & $36(67)$ & \\
\hline Brisk $(n=19)$ & $18(12)$ & $1(3)$ & & $18(11.8)$ & $1(3.7)$ & & 17 (13) & $2(4)$ & & $16(13)$ & $2(4)$ & \\
\hline
\end{tabular}

The number of observations, percentage shown in parentheses (\%), and the respective $P$-values are shown resultant from Pearson's $\chi^{2}$-test of association $\left(\chi^{2}\right)$, or Fisher's exact test, in which the frequency of observations in a cell was less than 5 (indicated with ${ }^{*}$ ). Statistically significant $P$-values are indicated by bold font. 
Table 2 The table shows the association between microvessel density, macrophage count, blood vessel invasion and haematoxylin and eosin-determined vascular invasion with clinicopathological variables in melanoma

\begin{tabular}{|c|c|c|c|c|c|c|c|c|c|c|c|c|}
\hline \multirow[t]{3}{*}{ Category } & \multicolumn{3}{|c|}{ Microvessel density } & \multicolumn{3}{|c|}{ Macrophage count } & \multicolumn{3}{|c|}{ Blood vessel invasion } & \multicolumn{3}{|c|}{ Vascular invasion } \\
\hline & \multicolumn{3}{|c|}{$\mathrm{n}(\%)$} & \multicolumn{3}{|c|}{$\mathrm{n}(\%)$} & \multicolumn{3}{|c|}{$n(\%)$} & \multicolumn{3}{|c|}{ Haematoxylin and eosin n (\%) } \\
\hline & Low & High & $\mathrm{P}$-value & Low & High & $\mathrm{P}$-value & Absent & Present & $\mathrm{P}$-value & Absent & Present & $\mathrm{P}$-value \\
\hline \multicolumn{13}{|l|}{ Gender } \\
\hline Female $(n=99)$ & $44(46)$ & $45(47)$ & \multirow[t]{2}{*}{0.88} & $51(54)$ & $44(44)$ & \multirow[t]{2}{*}{0.18} & $86(47)$ & $3(37)$ & \multirow[t]{2}{*}{$0.73^{*}$} & $90(49)$ & $6(37)$ & \multirow[t]{2}{*}{0.37} \\
\hline Male $(n=103)$ & $51(53)$ & $50(53)$ & & $44(46)$ & $56(56)$ & & $96(53)$ & $5(62)$ & & $93(51)$ & $10(62)$ & \\
\hline \multicolumn{13}{|c|}{ Sentinal node biopsy (SNB) } \\
\hline Negative $(n=70)$ & $38(40)$ & $25(26)$ & 0.05 & $32(34)$ & $36(36)$ & 0.73 & $61(34)$ & $2(25.0)$ & $1.00 *$ & $68(37)$ & $2(12)$ & $0.06^{*}$ \\
\hline Positive $(n=132)$ & $57(60)$ & $70(74)$ & & $63(66)$ & $64(64)$ & & $121(66)$ & $6(75.0)$ & & $115(63)$ & $14(87)$ & \\
\hline Relapse status & & & & & & & & & & & & \\
\hline No $(n=134)$ & $62(68)$ & $63(67)$ & 0.87 & $66(73)$ & $65(66)$ & 0.31 & $121(68)$ & $4(50)$ & $0.23^{*}$ & $126(71)$ & $8(62)$ & 0.09 \\
\hline Yes $(n=62)$ & $29(32)$ & $31(33)$ & & $25(27)$ & $34(34)$ & & $56(32)$ & $4(50)$ & & $52(29)$ & $5(38)$ & \\
\hline Staging & & & & & & & & & & & & \\
\hline $\mathrm{IA} / \mathrm{IB}(n=43)$ & $29(31)$ & $10(11)$ & $<0.001 *$ & $22(24)$ & $19(20)$ & $0.05^{*}$ & $39(22)$ & $0(0)$ & $0.47^{*}$ & $42(24)$ & $1(7)$ & $0.21^{*}$ \\
\hline IIA $(n=11)$ & $6(6)$ & $3(3)$ & & $5(5)$ & $6(6)$ & & $9(5)$ & $0(0)$ & & $10(6)$ & $1(7)$ & \\
\hline IIB/IIC $(n=12)$ & $2(2)$ & $9(10)$ & & $2(2)$ & $10(10)$ & & $10(6)$ & $1(14)$ & & $12(7)$ & $0(0)$ & \\
\hline IIIA $(n=88)$ & $44(47)$ & $40(44)$ & & $48(52)$ & $37(38)$ & & $80(45)$ & $4(57)$ & & $75(42)$ & $11(73)$ & \\
\hline IIIB $(n=41)$ & $12(13)$ & $28(31)$ & & $15(16)$ & $25(26)$ & & $38(22)$ & $2(29)$ & & $38(21)$ & $2(13)$ & \\
\hline Number of positive nod & & & & & & & & & & & & \\
\hline $0(n=70)$ & $38(40)$ & $25(26)$ & $0.06^{*}$ & $32(34)$ & $36(36)$ & $0.91^{*}$ & $61(34)$ & $2(25)$ & $0.41^{*}$ & $68(37)$ & $2(12)$ & $0.09^{*}$ \\
\hline 1 or $2(n=123)$ & $51(54)$ & $67(71)$ & & $59(62)$ & $59(59)$ & & $113(62)$ & $5(62)$ & & $107(58)$ & $13(81)$ & \\
\hline$\geqslant 3(n=9)$ & $6(6)$ & $3(3)$ & & $4(4)$ & $5(5)$ & & $8(4)$ & $1(12)$ & & $8(4)$ & $1(6)$ & \\
\hline Melanoma site & & & & & & & & & & & & \\
\hline Trunk $(n=73)$ & $34(36)$ & 37 (39) & 0.82 & $32(34)$ & 39 (39) & 0.19 & $67(37)$ & $4(50)$ & $0.38^{*}$ & $65(36)$ & $7(44)$ & $0.62^{*}$ \\
\hline $\operatorname{Leg}(n=67)$ & $34(36)$ & $28(29)$ & & $39(41)$ & $27(27)$ & & $59(32)$ & $3(37)$ & & $61(33)$ & $5(31)$ & \\
\hline $\operatorname{Arm}(n=45)$ & $20(21)$ & $23(24)$ & & $19(20)$ & $25(25)$ & & $43(24)$ & $0(0)$ & & $43(23)$ & $2(12)$ & \\
\hline Head/neck $(n=17)$ & $7(7)$ & $7(7)$ & & $5(5)$ & $9(9)$ & & $13(7)$ & $1(12)$ & & $14(8)$ & $2(12)$ & \\
\hline Histological subtype & & & & & & & & & & & & \\
\hline $\begin{array}{l}\text { Superficial spreading } \\
(n=141)\end{array}$ & $70(78)$ & $64(72)$ & $0.36^{*}$ & $73(81)$ & $63(67)$ & 0.03 & $130(75)$ & $4(67)$ & $0.37^{*}$ & $127(74)$ & $12(80)$ & $0.80^{*}$ \\
\hline Nodular $(n=37)$ & $14(16)$ & $21(24)$ & & $11(12)$ & $26(28)$ & & $34(20)$ & $1(17)$ & & $35(20)$ & $2(13)$ & \\
\hline Other $(n=12)$ & $6(7)$ & $4(4)$ & & $6(7)$ & $5(5)$ & & $9(5)$ & $1(17)$ & & $10(6)$ & $1(7)$ & \\
\hline Breslow thickness ( $\mathrm{mm}$ ) & & & & & & & & & & & & \\
\hline$\leqslant 1(n=94)$ & $53(56)$ & $34(36)$ & 0.02 & $55(58)$ & $34(34)$ & $<0.001$ & $84(46)$ & $3(37)$ & $0.89^{*}$ & $89(49)$ & $3(19)$ & $0.05^{*}$ \\
\hline$>1 \leqslant 2(n=79)$ & $32(34)$ & $42(44)$ & & $35(37)$ & $43(43)$ & & $70(38)$ & $4(50)$ & & $69(38)$ & $9(56)$ & \\
\hline$>2 \leqslant 4(n=29)$ & $10(11)$ & $19(20)$ & & $5(5)$ & $23(23)$ & & $28(15)$ & $1(12)$ & & $25(14)$ & $4(25)$ & \\
\hline Ulceration & & & & & & & & & & & & \\
\hline No $(n=145)$ & $80(87)$ & $54(59)$ & $<0.001$ & $79(85)$ & $60(62)$ & $<0.001$ & $130(74)$ & $4(57)$ & $0.39 *$ & $129(73)$ & $13(87)$ & $0.36^{*}$ \\
\hline Yes $(n=50)$ & $12(13)$ & $37(41)$ & & $14(15)$ & $36(37)$ & & $46(26)$ & $3(43)$ & & $48(27)$ & $2(13)$ & \\
\hline Mitotic rate (per $\mathrm{mm}^{2}$ ) & & & & & & & & & & & & \\
\hline$<3(n=68)$ & $41(43)$ & $21(22)$ & 0.002 & $36(38)$ & $27(27)$ & 0.005 & $61(34)$ & $1(12)$ & $0.29^{*}$ & $64(35)$ & 3 (19) & $0.03^{*}$ \\
\hline $3-7(n=62)$ & $30(32)$ & $28(30)$ & & $35(37)$ & $26(26)$ & & $56(31)$ & $2(25)$ & & $51(28)$ & $10(62)$ & \\
\hline$>7(n=71)$ & $24(25)$ & $25(48)$ & & $23(24)$ & $47(47)$ & & $64(35)$ & $5(62)$ & & $67(37)$ & 3 (19) & \\
\hline Perineural infiltration & & & & & & & & & & & & \\
\hline No $(n=186)$ & 87 (97) & 87 (100) & $0.25 *$ & $86(97)$ & $94(100)$ & $0.11^{*}$ & $168(98)$ & $6(100)$ & $1.00 *$ & $170(99)$ & $13(87)$ & $0.02^{*}$ \\
\hline Yes $(n=3)$ & $3(3)$ & $0(0)$ & & $3(3)$ & $0(0)$ & & $3(2)$ & $0(0)$ & & $1(1)$ & $2(13)$ & \\
\hline Regression & & & & & & & & & & & & \\
\hline No $(n=171)$ & $84(88)$ & $75(81)$ & 0.14 & $83(88)$ & $81(82)$ & 0.21 & $151(84)$ & $8(100)$ & 0.61 * & $155(85)$ & $13(87)$ & $1.00^{*}$ \\
\hline Yes $(n=29)$ & $11(12)$ & $18(19)$ & & $11(12)$ & $18(18)$ & & $29(16)$ & $0(0)$ & & 27 (15) & $2(13)$ & \\
\hline Microsatellites & & & & & & & & & & & & \\
\hline No $(n=163)$ & $79(88)$ & $74(86)$ & 0.73 & $78(88)$ & $80(86)$ & 0.75 & $149(88)$ & $4(67)$ & $0.18^{*}$ & $149(88)$ & $11(73)$ & $0.13^{*}$ \\
\hline Yes $(n=25)$ & $11(12)$ & $12(14)$ & & $11(12)$ & $13(14)$ & & 21 (12) & $2(33)$ & & 21 (12) & 4 (27) & \\
\hline Tumour infiltrating lyn & hocytes ( & TILs) & & & & & & & & & & \\
\hline Absent $(n=52)$ & $28(30)$ & $21(22)$ & 0.45 & $35(37)$ & $16(16)$ & 0.005 & $44(25)$ & $5(62)$ & 0.07 * & $48(27)$ & $4(27)$ & $0.59^{*}$ \\
\hline Non-brisk $(n=126)$ & $56(61)$ & $63(67)$ & & $51(54)$ & $71(73)$ & & $116(65)$ & $3(37)$ & & $113(63)$ & $11(73)$ & \\
\hline Brisk $(n=19)$ & $8(9)$ & $10(11)$ & & $8(9)$ & $10(10)$ & & $18(10)$ & $0(0.0)$ & & $19(11)$ & $0(0)$ & \\
\hline
\end{tabular}

The number of observations, percentage shown in parentheses (\%), and the respective $P$-values are shown resultant from Pearson's $\chi^{2}$-test of association $\left(\chi^{2}\right)$, or Fisher's exact test, in which the frequency of observations in a cell was less than 5 (indicated with *). Statistically significant $P$-values are indicated by bold font. 
and the presence of microsatellites $\left(\chi^{2}=7.69\right.$, d.f. $=1, P=0.006$ ). In addition, vascular invasion was also significantly associated with nodular histological subtype $\left(\chi^{2}=26.65\right.$, d.f. $=2, P<0.001$; Table 1).

\section{Distribution of Lymphatic and Blood Vessels in Cutaneous Melanoma}

Immunohistochemistry using anti-D2-40 antibody demonstrated consistent staining of lymphatic endothelial cells. Lymphatic vessels were observed in all peritumoural tissue, where this area was assessable $(100 \%, 181$ out of 181$)$. Intratumoural lymphatic vessels were observed in the majority of tumours (86\%, 165 out of 191). Peritumoural lymphatic vessel density ranged from 0.009-0.198 per $\mathrm{mm}^{2}$ (median 0.060 per $\mathrm{mm}^{2}$ ) and intratumoural lymphatic vessel density from $0.000-0.130$ per $\mathrm{mm}^{2}$ (median 0.010 per $\mathrm{mm}^{2}$ ). Total lymphatic density ranged from 0.004-0.266 per $\mathrm{mm}^{2}$ (median 0.077 per $\mathrm{mm}^{2}$ ) and microvessel density ranged from 0.6679.000 (median 4.833). Lymphatic vessel and microvessel density were not directly compared as different assessment methods were used; required due to the high frequency of blood vessels observed in comparison with the relatively low level of lymphatic vessels. ${ }^{24}$

\section{Relationship between Lymphatic and Microvessel Density and Clinicopathological Variables}

Low intratumoural lymphatic vessel density was not statistically associated with clinicopathological variables, but was marginally associated with relapse $\left(\chi^{2}=4.91\right.$, d.f. $\left.=1, P=0.03\right)$ and tumour infiltrating lymphocytes (TILs; $\chi^{2}=6.08$, d.f. $=2$ $P=0.05)$. Total lymphatic vessel density was significantly associated with the absence of TILs $\left(\chi^{2}=11.21\right.$, d.f. $=2, P=0.004$; Table 3$)$.

High microvessel density was associated with poor prognostic histological factors, being more frequent in the presence of ulceration $\left(\chi^{2}=17.79\right.$, d.f. $=1, P<0.001)$ and high mitotic rate $\left(\chi^{2}=12.91\right.$, d.f. $=2, P=0.002$ ), and was positively associated with stage $\left(\chi^{2}=12.91\right.$, d.f. $\left.=2, P<0.001\right)$. Furthermore high microvessel density was marginally positively associated with positive sentinel node biopsy $\left(\chi^{2}=4.01\right.$, d.f. $=1, P=0.05$; Table 2$)$.

\section{Relationship between Macrophage Count and Clinicopathological Data}

The presence of tumour infiltrating macrophages was investigated using anti-CD68 antibody, which is able to detect both polarised type 1 (M1) and type 2 (M2) macrophages resulting from alternate differentiation. ${ }^{28}$ High macrophage count was associated with the presence of ulceration $\left(\chi^{2}=12.23\right.$, d.f. $=1$,
$P<0.001)$, Breslow thickness $\left(\chi^{2}=17.23\right.$, d.f. $=2$, $P<0.001)$, mitotic rate $\left(\chi^{2}=10.67, \quad\right.$ d.f. $=2$, $P=0.005)$ and the presence of non-brisk or brisk TILs $\left(\chi^{2}=10.53\right.$, d.f. $\left.=2, P=0.005\right)$. No other associations with clinicopathological variables were observed (Table 2).

\section{Relationship between Vessel Density, Invasion and Macrophage Count}

Intratumoural lymphatic density was associated with intratumoural lymphatic vessel invasion $\left(\chi^{2}=8.05\right.$, d.f. $\left.=1, P=0.005\right)$, but not peritumoural invasion. Blood microvessel density was associated with intratumoural, peritumoural and any lymphatic invasion $\left(\chi^{2}=8.08\right.$, d.f. $=1, P=0.004 ; \chi^{2}=6.65$, d.f. $=1, \quad P=0.010 ; \quad \chi^{2}=14.77$, d.f. $=, 1 \quad P<0.001$, respectively). A high macrophage count was significantly associated with the presence of any lymphatic vessel invasion $\left(\chi^{2}=9.15\right.$, d.f. $=1$, $P=0.002)$, blood microvessel density $\left(\chi^{2}=8.94\right.$, d.f. $=1, \quad P=0.003)$ and immunohistochemically determined vascular invasion $\left(\chi^{2}=8.66\right.$, d.f. $=1$, $P=0.003$ ), indicating the likely importance of macrophage in the process of tumour vascularisation and lymphatic dissemination.

\section{Clinical Outcome and Vessel Density, Invasion and Macrophage Involvement}

None of the variables assessed in this study were statistically associated with relapse-free or overall survival in univariate analysis, and were therefore not assessed in multivariate analysis.

\section{Discussion}

This study analysed vessel invasion and density, both blood and lymphatic in primary melanomas as prognostic markers. In addition, the contribution of tumour-associated macrophages to this process was evaluated. To our knowledge, this is one of the largest studies of its type so far, which benefits from having a large proportion of positive sentinel node biopsy patients compared with previous studies, and also from a single expert melanoma pathologist' review of the primary melanoma and sentinel node biopsy pathology.

Discrepancies exist between vascular invasion determined by immunohistochemistry and H\&E, principally due to difficulties in determining the vessel endothelium surrounding tumour emboli or confounding fixation-related artefacts in H\&Estained tissues. The detection rates of vascular invasion determined in H\&E-stained tissues in comparison with lymphatic vessel invasion determined by immunohistochemistry in previously published studies have ranged from $0-16,{ }^{29} 0-17,{ }^{15}$ $0-23,{ }^{9} 3.1-21.9^{13}$ and $4-37 \% .{ }^{14}$ In the current study, 
Table 3 The table shows the association between lymphatic density measures and clinicopathological variables in melanoma

\begin{tabular}{|c|c|c|c|c|c|c|c|c|c|}
\hline \multirow[t]{3}{*}{ Category } & \multicolumn{9}{|c|}{ Lymphatic density } \\
\hline & \multicolumn{3}{|c|}{ Intratumoural n (\%) } & \multicolumn{3}{|c|}{ Peritumoural n (\%) } & \multicolumn{3}{|c|}{ Total n (\%) } \\
\hline & Low & High & $\mathrm{P}$-value & Low & High & $\mathrm{P}$-value & Low & High & P-value \\
\hline \multicolumn{10}{|l|}{ Gender } \\
\hline Female $(n=99)$ & $48(51)$ & $44(46)$ & 0.52 & $45(51)$ & $44(48)$ & 0.71 & $50(52)$ & $42(44)$ & 0.28 \\
\hline Male $(n=103)$ & $47(49)$ & $52(54)$ & & $44(49)$ & $48(52)$ & & $46(48)$ & $53(56)$ & \\
\hline \multicolumn{10}{|l|}{ Sentinal node biopsy (SNB) } \\
\hline Negative $(n=70)$ & $38(40)$ & $28(29)$ & 0.12 & $35(39)$ & $31(34)$ & 0.43 & $36(37)$ & $30(32)$ & 0.39 \\
\hline Positive $(n=132)$ & $57(60)$ & $68(71)$ & & $54(61)$ & $61(66)$ & & $60(62)$ & $65(68)$ & \\
\hline \multicolumn{10}{|l|}{ Relapse status } \\
\hline No $(n=134)$ & $57(61)$ & $71(76)$ & 0.03 & $61(70)$ & $63(69)$ & 0.90 & $61(66)$ & $67(72)$ & 0.34 \\
\hline Yes $(n=62)$ & $36(39)$ & $22(24)$ & & $26(30)$ & $91(31)$ & & $32(34)$ & $26(28)$ & \\
\hline \multicolumn{10}{|l|}{ Staging } \\
\hline IA/IB $(n=43)$ & $23(26)$ & $17(18)$ & 0.69 & $23(27)$ & $17(19)$ & 0.58 & $22(24)$ & $18(19)$ & 0.78 \\
\hline IIA $(n=11)$ & $6(7)$ & $5(5)$ & & $6(7)$ & $5(6)$ & & $6(7)$ & $5(5)$ & \\
\hline IIB/IIC $(n=12)$ & $6(7)$ & $5(5)$ & & $5(6)$ & $6(7)$ & & $5(5)$ & $6(6)$ & \\
\hline IIIA $(n=88)$ & $38(42)$ & $45(48)$ & & $33(38)$ & $44(50)$ & & $37(41)$ & $46(49)$ & \\
\hline IIIB $(n=41)$ & $17(19)$ & $22(23)$ & & $19(22)$ & $16(18)$ & & $21(23)$ & $18(19)$ & \\
\hline \multicolumn{10}{|l|}{ Number of positive nodes } \\
\hline $0(n=70)$ & $38(40)$ & $28(29)$ & $0.31^{*}$ & $35(39)$ & $31(34)$ & $0.35^{*}$ & $36(37)$ & $30(32)$ & $0.37^{*}$ \\
\hline 1 or $2(n=123)$ & $53(56)$ & $63(66)$ & & $48(54)$ & $58(63)$ & & $54(56)$ & $62(65)$ & \\
\hline$\geqslant 3(n=9)$ & $4(4)$ & $5(5)$ & & $6(7)$ & $3(3)$ & & $6(6)$ & $3(3)$ & \\
\hline \multicolumn{10}{|l|}{ Melanoma site } \\
\hline Trunk $(n=73)$ & $40(42)$ & $32(33)$ & 0.27 & $35(39)$ & $32(35)$ & 0.44 & $35(36)$ & 37 (39) & 0.12 \\
\hline $\operatorname{Leg}(n=67)$ & $25(26)$ & $38(40)$ & & $30(34)$ & $29(32)$ & & $35(36)$ & $28(29)$ & \\
\hline Arm $(n=45)$ & $23(24)$ & $19(20)$ & & $20(22)$ & $21(23)$ & & $23(24)$ & $19(20)$ & \\
\hline Head/neck $(n=17)$ & $7(7)$ & $7(7)$ & & $4(4)$ & $10(11)$ & & $3(3)$ & $11(12)$ & \\
\hline \multicolumn{10}{|l|}{ Histological subtype } \\
\hline Superficial spreading $(n=141)$ & $65(75)$ & $70(75)$ & $0.21^{*}$ & $68(79)$ & $64(74)$ & 0.72 & $67(75)$ & $68(75)$ & $0.55^{*}$ \\
\hline Nodular $(n=37)$ & $14(16)$ & $20(22)$ & & $13(15)$ & $17(20)$ & & $15(17)$ & $19(21)$ & \\
\hline Other $(n=12)$ & $8(9)$ & $3(3)$ & & $5(6)$ & $5(6)$ & & $7(8)$ & $4(4)$ & \\
\hline \multicolumn{10}{|l|}{ Breslow thickness (mm) } \\
\hline$\leqslant 1(n=94)$ & $48(51)$ & $40(42)$ & 0.08 & $44(49)$ & $43(47)$ & 0.83 & $46(48)$ & $42(44)$ & 0.13 \\
\hline$>1 \leqslant 2(n=79)$ & $30(32)$ & $45(47)$ & & $32(36)$ & $37(40)$ & & $32(33)$ & $43(45)$ & \\
\hline$>2 \leqslant 4(n=29)$ & $17(18)$ & $11(12)$ & & $13(15)$ & $12(13)$ & & $18(19)$ & $10(11)$ & \\
\hline \multicolumn{10}{|l|}{ Ulceration } \\
\hline No $(n=145)$ & $69(77)$ & $67(71)$ & 0.41 & $66(76)$ & $64(74)$ & 0.73 & $69(75)$ & $67(73)$ & 0.74 \\
\hline Yes $(n=50)$ & $21(23)$ & $27(29)$ & & $21(24)$ & $23(26)$ & & $23(25)$ & $25(27)$ & \\
\hline \multicolumn{10}{|l|}{ Mitotic rate (per $\mathrm{mm}^{2}$ ) } \\
\hline$<3(n=68)$ & $38(40)$ & $26(27)$ & 0.15 & $36(41)$ & $26(28)$ & 0.20 & $39(41)$ & $25(26)$ & 0.08 \\
\hline $3-7(n=62)$ & $26(28)$ & $32(33)$ & & $24(27)$ & $31(34)$ & & $24(25)$ & $34(36)$ & \\
\hline$>7(n=71)$ & $30(32)$ & $38(40)$ & & $28(32)$ & $35(38)$ & & $32(34)$ & $36(38)$ & \\
\hline \multicolumn{10}{|l|}{ Perineural infiltration } \\
\hline No $(n=186)$ & $85(97)$ & $90(100)$ & $0.12^{*}$ & $81(96)$ & $85(100)$ & $0.12^{*}$ & $86(97)$ & 89 (100) & $0.25^{*}$ \\
\hline Yes $(n=3)$ & $3(3)$ & $0(0)$ & & $3(4)$ & $0(0)$ & & $3(3)$ & $0(0)$ & \\
\hline \multicolumn{10}{|l|}{ Regression } \\
\hline No $(n=171)$ & $78(84)$ & $82(85)$ & 0.77 & $75(86)$ & $75(82)$ & 0.40 & $81(86)$ & $79(83)$ & 0.57 \\
\hline Yes $(n=29)$ & $15(16)$ & $14(15)$ & & $12(14)$ & $17(18)$ & & $13(14)$ & $16(17)$ & \\
\hline Microsatellites & & & & & & & & & \\
\hline No $(n=163)$ & $76(86)$ & 79 (89) & 0.63 & $69(83)$ & $78(92)$ & 0.09 & $74(84)$ & $81(91)$ & 0.16 \\
\hline Yes $(n=25)$ & $12(14)$ & $10(11)$ & & $14(17)$ & $7(8)$ & & $14(16)$ & $8(9)$ & \\
\hline Tumour infiltrating lymphocytes & & & & & & & & & \\
\hline Absent $(n=52)$ & $32(34)$ & $18(19)$ & 0.05 & $28(32)$ & $19(21)$ & 0.21 & $35(38)$ & $15(16)$ & 0.004 \\
\hline Non-brisk $(n=126)$ & $54(58)$ & $64(68)$ & & $51(59)$ & $62(67)$ & & $50(54)$ & $68(72)$ & \\
\hline Brisk $(n=19)$ & $7(8)$ & $12(13)$ & & $8(9)$ & $11(12)$ & & $8(9)$ & $11(12)$ & \\
\hline
\end{tabular}

The number of observations, percentage shown in parentheses (\%), and the respective $P$-values are shown resultant from Pearson's $\chi^{2}$-test of association $\left(\chi^{2}\right)$, or Fisher's exact test, in which the frequency of observations in a cell was less than 5 (indicated with *). Statistically significant $P$-values are indicated by bold font. 
an increase from $8-30 \%$ total vascular (both lymphatic and blood) invasion was observed, or $27 \%$, if only lymphatic vessel invasion is considered. Previous work and the data presented here show that assessment of vascular invasion using conventional H\&E-stained tissue is fraught with difficulty, with a high false positive and negative detection rate.

Reported detection rates for lymphatic vessel invasion are $16,{ }^{12,29} 17,,^{15} 21.9,{ }^{13} 23,{ }^{9} 33,{ }^{10,11} 37,{ }^{14}$ $47^{6}$ and $63 \%^{7}$ in melanoma. In this study, we demonstrated lymphatic vessel invasion in $27 \%$ of cases. A preference of lymphatic over blood vessel invasion has been reported previously with varying frequencies of blood vessel to lymphatic vessel invasion of $3-16,{ }^{12} 4.7-33^{11}$ and $43-47 \%,{ }^{6}$ respectively; our current study demonstrates that lymphatic vessel invasion occurs more frequently than blood vessel invasion (27 vs 4\%). In addition, three of the eight cases identified as having blood vessel invasion also had lymphatic vessel invasion. D2-40 was used to stain lymphatic vessels as it stains lymphatic cells, but also some other cell types such as mesothelial and ependymal cells; although prospero-related homeobox gene-1 (prox-1) specifically stains lymphatic cells, it stains only the lymphatic nuclei, making interpretation more difficult, and VEGFR-3 is expressed on angiogenic blood vessels in tumours. ${ }^{30}$

It is unclear as to what would cause preferential lymphatic invasion, in tumours with a rich vascular network. Interestingly, dermal lymphatic endothelial cells, not blood endothelial cells, express SLC/ CCL21 the ligand for the CC chemokine receptor 7 expressed on melanoma cells, and this ligand/ receptor interaction is implicated in the recruitment of mature dendritic cells from interstitial spaces to lymphatic vessels. ${ }^{31}$ In-vivo murine models have suggested that the intratumoural lymphatic network may be non-functional and lymphatic vessels at the tumour margin may be enough for metastasis. ${ }^{32}$

Previous studies have demonstrated an association between intratumoural lymphatic vessel density and sentinel node biopsy positivity (37 patients; $;^{33} 45$ patients $^{5}$ ). Furthermore, the use of lymphatic density has been incorporated into the Shield's Index, also including lymphatic vessel invasion and Breslow thickness, which can be used to predict metastasis in melanoma patients. ${ }^{6,7}$ In the current study, no associations were observed with either lymphatic vessel density, or microvessel density and clinical outcome; however, microvessel density was associated with markers of more aggressive disease including ulceration, tumour stage and mitotic rate. Studies that have previously investigated lymphatic vessel density in melanoma have focussed on investigating associations with clinical outcome; of those that have examined links with clinicopathological variables associations between lymphatic vessel density and age, ${ }^{3,5}$ the presence of inflammatory infiltrate, ${ }^{5,8}$ positive sentinel lymph node, ${ }^{5}$ tumour stage ${ }^{4}$ and tumour thickness ${ }^{3}$ have been described. Previous studies have not shown an association between lymphatic vessel density and ulceration. ${ }^{3,5,6}$ Associations between microvessel density and Clark's level ${ }^{4}$ and tumour stage ${ }^{4}$ have also been previously reported.

The presence of a high macrophage count was significantly associated with the presence of any lymphatic vessel invasion, high microvessel density and total immunohistochemically determined vascular invasion. Previous studies have highlighted associations between VEGF-C, an activator of lymphangiogenesis and lymphatic vessel density, and it may be possible that a putative macrophage effect on vessels might be mediated in part through this growth factor. ${ }^{33,34}$ VEGF-C expression by the tumour has been shown to increase lymphangiogenesis in in-vivo murine models, ${ }^{32,35}$ but also associated with the recruitment macrophages via the VEGFR-3 receptor, which is also found on the surface of lymphatic vessels. ${ }^{35}$ In addition to VEGF-C, there may be a number of cytokines involved in promoting lymphatic vessel invasion as observed in in-vitro studies. Nitric oxide (NO) has been implicated in the process of lymphangiogenesis, and the expression of inducible NO synthase in melanoma is associated with lymphatic vessel density. ${ }^{36}$ Interestingly, a study by the same group suggested that inducible NO synthase expressed by tumour-associated macrophages may contribute to elevated $\mathrm{NO}^{37}$ Jensen et $a l^{20}$ investigated the presence of CD68-stained macrophages at the invasive front of melanoma tumours, in tumour nests and in tumour stroma, and showed an association with poor overall survival and linked the presence of macrophages in tumour nests and stroma to patient age and median thickness. No association with the presence of macrophages and clinical outcome was observed in the current study; however, there was a significant association between a high macrophage count and ulceration, Breslow thickness, mitotic rate and tumour infiltrating lymphocytes. Molecular characterisation of sentinel nodes, with and without tumour metastasis, and non-sentinel nodes, revealed an elevated gene expression of interleukin 13 , leptin, lymphotoxin $\beta$-receptor and macrophage inflammatory protein $1 \mathrm{~b}$, highlighting the role of cytokine profile in subsequent nodal metastasis, in particular, MIP1B which has been shown to increase invasion of uveal melanoma. ${ }^{38,39}$

In conclusion, data from the current study shows that in melanoma, vascular invasion occurs predominantly via lymphatic vessels. Furthermore, the data demonstrate that vascular invasion is more accurately detected in immunohistochemistrystained-tissue using specific endothelial markers, than conventional assessment in H\&E-stained tissue. Vascular invasion was associated with factors indicative of a poor prognosis including stage, increased Breslow thickness and ulceration. Lymphatic vessel density was associated with microvessel density, and macrophage count was 
associated with ulceration and mitotic rate. Microvessel density, lymphatic vessel density, lymphatic and blood vessel invasion were not associated with relapse-free or overall survival. The observation that vessel characteristics investigated were not associated with relapse-free or overall survival was unexpected due to their strong association with known adverse prognostic histological features of primary melanoma; however, this may attest to the value of existing histological biomarkers. Because of the retrospective nature of the data collection, the follow-up time of this study was not standardised and varied from 1 day to 111.7 months, which is a limitation of this study. Perhaps, most interestingly, these results suggest that a high macrophage count is associated with markers of neovascularisation and corresponding primary tumour growth, and the suggestion is therefore that macrophages may have a role in vascular neogenesis. A high macrophage count may also have a role in cancer cell dissemination by regulating preferential invasion of lymphatic over blood vessels, as an association was observed between lymphatic vessel invasion and macrophage count.

\section{Acknowledgements}

We would like to acknowledge British Skin Foundation (grant number S401) for funding this work. S Safuan is funded by the Ministry of Higher Education, Malaysia, and the Science University of Malaysia. FE, CW and JAN-B are supported by Cancer Research UK (Project grants C8216/A6129 and C8216/A8168, and Programme grant C588/ A4994). AM was supported by a clinical fellowship awarded by the Special Trustees of the Leeds Teaching Hospitals Trust.

\section{Disclosure/conflict of interest}

The authors declare no conflict of interest.

\section{References}

1 Balch CM, Gershenwald JE, Soong SJ, et al. Final version of 2009 AJCC melanoma staging and classification. J Clin Oncol 2009;27:6199-6206.

2 Mohammed RA, Martin SG, Gill MS, et al. Improved methods of detection of lymphovascular invasion demonstrate that it is the predominant method of vascular invasion in breast cancer and has important clinical consequences. Am J Surg Pathol 2007;31: 1825-1833.

3 Straume O, Jackson DG, Akslen LA. Independent prognostic impact of lymphatic vessel density and presence of low-grade lymphangiogenesis in cutaneous melanoma. Clin Cancer Res 2003;9:250-256.

4 Valencak J, Heere-Ress E, Kopp T, et al. Selective immunohistochemical staining shows significant prognostic influence of lymphatic and blood vessels in patients with malignant melanoma. Eur J Cancer 2004; 40:358-364.

5 Massi D, Puig S, Franchi A, et al. Tumour lymphangiogenesis is a possible predictor of sentinel lymph node status in cutaneous melanoma: a case-control study. J Clin Pathol 2006;59:166-173.

6 Shields JD, Borsetti M, Rigby H, et al. Lymphatic density and metastatic spread in human malignant melanoma. Br J Cancer 2004;90:693-700.

7 Emmett MS, Symonds KE, Rigby H, et al. Prediction of melanoma metastasis by the Shields index based on lymphatic vessel density. BMC Cancer 2010;10:208.

8 Dadras SS, Paul T, Bertoncini J, et al. Tumor lymphangiogenesis: a novel prognostic indicator for cutaneous melanoma metastasis and survival. Am J Pathol 2003;162:1951-1960.

9 Petersson F, Diwan AH, Ivan D, et al. Immunohistochemical detection of lymphovascular invasion with D2-40 in melanoma correlates with sentinel lymph node status, metastasis and survival. J Cutan Pathol 2009;36:1157-1163.

10 Niakosari F, Kahn HJ, McCready D, et al. Lymphatic invasion identified by monoclonal antibody D2-40, younger age, and ulceration: predictors of sentinel lymph node involvement in primary cutaneous melanoma. Arch Dermatol 2008;144:462-467.

11 Xu X, Gimotty PA, Guerry D, et al. Lymphatic invasion revealed by multispectral imaging is common in primary melanomas and associates with prognosis. Hum Pathol 2008;39:901-909.

12 Doeden K, Ma Z, Narasimhan B, et al. Lymphatic invasion in cutaneous melanoma is associated with sentinel lymph node metastasis. J Cutan Pathol 2009;36:772-780.

13 Fohn LE, Rodriguez A, Kelley MC, et al. D2-40 lymphatic marker for detecting lymphatic invasion in thin to intermediate thickness melanomas: association with sentinel lymph node status and prognostic valuea retrospective case study. J Am Acad Dermatol 2011;64:336-345.

14 Petitt M, Allison A, Shimoni T, et al. Lymphatic invasion detected by D2-40/S-100 dual immunohistochemistry does not predict sentinel lymph node status in melanoma. J Am Acad Dermatol 2009;61:819-828.

15 Sahni D, Robson A, Orchard G, et al. The use of LYVE1 antibody for detecting lymphatic involvement in patients with malignant melanoma of known sentinel node status. J Clin Pathol 2005;58:715-721.

16 Kashani-Sabet M, Sagebiel RW, Ferreira CM, et al. Vascular involvement in the prognosis of primary cutaneous melanoma. Arch Dermatol 2001;137: 1169-1173.

17 Vuylsteke RJ, van Leeuwen PA, Statius Muller MG, et al. Clinical outcome of stage I/II melanoma patients after selective sentinel lymph node dissection: long-term follow-up results. J Clin Oncol 2003;21: 1057-1065.

18 Nagore E, Oliver V, Botella-Estrada R, et al. Prognostic factors in localized invasive cutaneous melanoma: high value of mitotic rate, vascular invasion and microscopic satellitosis. Melanoma Res 2005;15: 169-177.

19 Paek SC, Griffith KA, Johnson TM, et al. The impact of factors beyond Breslow depth on predicting sentinel lymph node positivity in melanoma. Cancer 2007;109: 100-108.

20 Jensen TO, Schmidt H, Moller HJ, et al. Macrophage markers in serum and tumor have prognostic impact in 
American Joint Committee on Cancer stage I/II melanoma. J Clin Oncol 2009;27:3330-3337.

21 Shi L, Lei D, Ma C, et al. Clinicopathological implications of tumour-associated macrophages and vascularization in sinonasal melanoma. J Int Med Res 2010; 38:1276-1286.

22 Rofstad EK, Mathiesen B. Metastasis in melanoma xenografts is associated with tumor microvascular density rather than extent of hypoxia. Neoplasia 2010; 12:889-898.

23 Mitra A, Conway C, Walker C, et al. Melanoma sentinel node biopsy and prediction models for relapse and overall survival. Br J Cancer 2010;103:1229-1236.

24 Mohammed RA, Martin SG, Mahmmod AM, et al. Objective assessment of lymphatic and blood vascular invasion in lymph node-negative breast carcinoma: findings from a large case series with long-term followup. J Pathol 2011;223:358-365.

25 Ammar A, Mohammed RA, Salmi M, et al. Lymphatic expression of CLEVER-1 in breast cancer and its relationship with lymph node metastasis. Anal Cell Pathol (Amst) 2011;34:67-78.

26 Fox SB, Harris AL. Histological quantitation of tumour angiogenesis. APMIS 2004;112:413-430.

27 Mohammed RA, Ellis IO, Elsheikh S, et al. Lymphatic and angiogenic characteristics in breast cancer: morphometric analysis and prognostic implications. Breast Cancer Res Treat 2009;113:261-273.

28 Pulford KA, Rigney EM, Micklem KJ, et al. KP1: a new monoclonal antibody that detects a monocyte/macrophage associated antigen in routinely processed tissue sections. J Clin Pathol 1989;42:414-421.

29 Niakosari F, Kahn HJ, Marks A, et al. Detection of lymphatic invasion in primary melanoma with monoclonal antibody D2-40: a new selective immunohistochemical marker of lymphatic endothelium. Arch Dermatol 2005;141:440-444.

30 Mohammed RA, Ellis IO, Lee AH, Martin SG. Vascular invasion in breast cancer; an overview of recent prognostic developments and molecular pathophysiological mechanisms. Histopathology 2009;55:1-9.

31 Kriehuber E, Breiteneder-Geleff S, Groeger M, et al. Isolation and characterization of dermal lymphatic and blood endothelial cells reveal stable and functionally specialized cell lineages. J Exp Med 2001;194:797-808.

32 Padera TP, Kadambi A, di Tomaso E, et al. Lymphatic metastasis in the absence of functional intratumor lymphatics. Science 2002;296:1883-1886.

33 Dadras SS, Lange-Asschenfeldt B, Velasco P, et al. Tumor lymphangiogenesis predicts melanoma metastasis to sentinel lymph nodes. Mod Pathol 2005;18:1232-1242.

34 Boone B, Blokx W, De Bacquer D, et al. The role of VEGF-C staining in predicting regional metastasis in melanoma. Virchows Arch 2008;453:257-265.

35 Skobe M, Hamberg LM, Hawighorst T, et al. Concurrent induction of lymphangiogenesis, angiogenesis, and macrophage recruitment by vascular endothelial growth factor-C in melanoma. Am J Pathol 2001;159: 893-903.

36 Massi D, De Nisi MC, Franchi A, et al. Inducible nitric oxide synthase expression in melanoma: implications in lymphangiogenesis. Mod Pathol 2009;22:21-30.

37 Massi D, Marconi C, Franchi A, et al. Arginine metabolism in tumor-associated macrophages in cutaneous malignant melanoma: evidence from human and experimental tumors. Hum Pathol 2007;38:1516-1525.

38 Torisu-Itakura H, Lee JH, Scheri RP, et al. Molecular characterization of inflammatory genes in sentinel and nonsentinel nodes in melanoma. Clin Cancer Res 2007;13:3125-3132.

39 Woodward JK, Elshaw SR, Murray AK, et al. Stimulation and inhibition of uveal melanoma invasion by HGF, GRO, IL-1alpha and TGF-beta. Invest Ophthalmol Vis Sci 2002;43:3144-3152.

(c) MUER RIEHISBREST Commons Attribution-NonCommercialNo Derivative Works 3.0 Unported License. To view a copy of this license, visit http://creativecommons.org/licenses/by-nc-nd/3.0/ 